\title{
Journal summary
}

\section{Leader}

Antiphospholipid syndrome

p 355

There is a very strong association between antiphospholipid antibodies and thrombosis: the presence of these antibodies is a risk factor in obstetrics as well as in general medicine and rheumatology. Valvular heart disease is increasingly recognised as occurring in association with them as well as thrombosis, and clinically the syndrome is relatively common. Three papers appear elsewhere in this issue (pages 358-61, 362-7, 430-1) describing features of the antiphospholipid syndrome, and this leader weaves a common thread between them.

\section{Scientific papers}

Antiphospholipid antibodies and occlusive vascular disease in systemic lupus erythematosus p 358 This is one of the papers referred to in the leader and draws attention to occlusive vascular disease in systemic lupus erythematosus and the presence of anticardiolipin antibodies.

Primary antiphospholipid syndrome p 362 More on a similar theme, this time from the Royal Postgraduate Medical School at Hammersmith, describing the features of the primary syndrome and drawing attention to the fact that although it may be related to systemic lupus erythematosus and other autoimmune diseases, it exists as a syndrome in its own right.

\section{NSAIDs and their effect on alkaline}

\section{phosphatase in arthritis}

The alkaline phosphatase concentration has been noted to rise in the presence of constrictive pericarditis. This study showed that non-steroidal antiinflammatory drugs have no effect on alkaline phosphatase concentrations in rheumatoid arthritis, osteoarthritis, or polymyalgia rheumatica.

\section{Antiarthritic drugs and proteoglycan} secretion

This Australian study describes a new method for examining the secretion of proteoglycans from rabbit chondrocytes, and the effect of antiarthritic drugs on this process. The inhibition of this secretion was variable, and interestingly the relation to drug concentration was also important. Is this going to be a more useful way of screening antiarthritic drugs in the future?

\section{Anti-inflammatory properties of} hydroxypyridinones p 382

Professor Blake has long been interested in the effect of synovial iron deposition in rheumatoid arthritis. Here, he and his coworkers explore this potential tissue damage effect further.

Sulphasalazine and hydroxychloroquine

in rheumatoid arthritis

Both these drugs are commonly used as second line agents in the treatment of rheumatoid arthritis. This double blind study compares them and finds little to choose between them, except that the therapeutic effect of sulphasalazine starts earlier.

\section{Aminohydroxypropylidene bisphosphonate} treatment in rheumatoid arthritis p 396 Osteoporosis in rheumatoid arthritis is an important feature. This Scottish study looked at aminohydroxypropylidene bisphosphonate in the treatment of this disease. Although it had a general effect on the skeleton as a whole, it had little influence on periarticular bone changes. This differential effect is intriguing.

\section{Synovial fluid in rheumatoid arthritis}

and $B$ cell factors

The pathogenesis of rheumatoid arthritis is complex, as the authors of this paper point out. This has led them to direct their interest to the presence of immunoregulatory B cell factors in synovial fluid, and they describe their conclusions.

DR4 prevalence and age of onset of rheumatoid arthritis

HLA-DR4 and its relation with rheumatoid arthritis is well known. Does this prevalence have any association with age at onset of the disease? Apparently not.

Haemodialysis and arthritic disease p 409 Haemodialysis is now a common and well established treatment for chronic renal failure. The development of significant arthritis and bone disease in these patients is of increasing interest, and the deposition of $\beta_{2}$ microglobulin amyloid increases with the 
duration of dialysis. Perhaps alteration of technique may modify this.

Childhood infection and Behçet's disease p 421 A variety of features in childhood seem to be associated with an increased risk of getting this disease. Sexual intercourse before the age of 16 years seems to be one of them.

Systemic lupus erythematosus and thyrotoxicosis

The coexistence of these two conditions usually passes unnoticed, but here the hyperthyroidism may just be a presentation of thyroiditis.

\section{Rapid report}

Monoclonal antibody, $T$ cells, and rheumatoid arthritis p 428

This is the first time that the facility of a rapid report with accelerated publication has been used in the Annals. The report describes the beneficial effect of a monoclonal antibody in rheumatoid arthritis resistant to treatment. Is this a portent for thệ future?

\section{Case reports}

The anticardiolipin syndrome and acute adrenal insufficiency

This is the third of the papers in this issue concerno ing the antiphospholipid syndrome, and is the first report of its association with acute adrenal gland failure.

\section{Adjuvant mycobacterial treatment for carcinoma of the bladder}

Local BCG treatment is now used for the treatmenit of many neoplasms-here it seems to have triggeresp off a reactive arthritis. The implications of this are greater than they might at first seem.

\section{IgA nephropathy and ankylosing} spondylitis

Another first-this time IgA nephropathy in woman with ankylosing spondylitis. It has beefs previously recognised in men, however. 